\title{
DRUG POLYMORPHISM IDENTIFICATION USING FOURIER TRANSFORM-RAMAN SPECTROSCOPY: A COMPARATIVE STUDY OF LAMIVUDINE AND FINASTERIDE DRUGS
}

\author{
CHANDRA SEKHARA RAO $\mathbf{M}^{1,2}$, CHENNA KRISHNA REDDY R $^{2,3}$, CHANDRA SEKHAR KB ${ }^{2}$, RAMI REDDY YV ${ }^{1 *}$
}

${ }^{1}$ Department of Chemistry, Sri Venkateswara University, Tirupati, Andhra Pradesh, India. ${ }^{2}$ Department of Chemistry, Jawaharlal Nehru Technological University Anantapur, Ananthapuramu, Andhra Pradesh, India. ${ }^{3}$ Department of Science and Humanities, Sri Venkateswara College of Engineering, Tirupati, Andhra Pradesh, India. *Email: dryvrsvu@gmail.com

Received: 28 September 2020, Revised and Accepted: 12 November 2020

ABSTRACT

Objectives: Maintaining the quality of the pharmaceutical drug product during its shelf life is highly desirable. The crystalline form of the drug having the great thermodynamic stability is essential for the manufacturers in pharmaceutical industry in view of their profit and also for the safety of the customer. Many pharmaceutical drugs have the tendency to exhibit polymorphism which is unwanted for pharmaceutical companies, where they have experienced market shortages due to these unpredicted polymorphic and/or pseudomorphic changes. The property of a drug exhibiting more than one crystal form is considerably regarded as polymorphism and each of the crystalline form has its own physicochemical properties, namely, solubility, heat capacity, melting point, and sublimation point. To relieve this ultimate effect on the drug quality and stability, a prior detection of polymorphism in the final dosage form is highly recommended. Hence, many analytical techniques have been proposed for the detection of polymorphism in pharmaceutical drug products.

Methods: Fourier transform (FT)-Raman spectrometer is used for the investigation of drug polymorphism and the instrument is advanced with charge coupled device detectors, ease of sample preparation and handling, mitigation of sub-sampling problems using different geometric laser irradiance patterns and having different optical components of Raman spectrometers.

Results: In this work, we carefully studied the Raman spectral patterns for Lamivudine as well as Finasteride drug substances for the detection of polymorphism. Further, we have highlighted the advantages of FT-Raman spectroscopy over other polymorphism detection techniques. For example, Raman spectra showed invariably sharp, well resolved bands compare to IR spectra due to the minor contribution of overtone vibrations in Raman spectra, resulting in much less broadening and a better resolution of bands. Besides, Raman spectroscopy does not suffer from the sampling problems that are common in X-ray powder diffraction, where preferred orientation and specimen displacements are serious restrictions for the application of quantitative method.

Conclusion: Here, in this paper, we are presented and compared the experimental results regarding the detection of polymorphism in Lamivudine and Finasteride drugs using FT-Raman spectroscopy, to illustrate the advantages of the technique in the detection of polymorphism over other techniques.

Keywords: Polymorphism, Comparison, Finasteride, Lamivudine, Fourier Transform-Raman spectroscopy.

(c) 2021 The Authors. Published by Innovare Academic Sciences Pvt Ltd. This is an open access article under the CC BY license (http://creativecommons.org/ licenses/by/4.0/) DOI: http://dx.doi.org/10.22159/ajpcr.2021v14i1.39881. Journal homepage: https://innovareacademics.in/journals/index.php/ajpcr

\section{INTRODUCTION}

Commercialization of pharmaceutical products involves a lengthy and time consuming process because (i) the product has to be tested for its suitability toward the target application, (ii) safety, (iii) efficacy, and (iv) need to conduct customer satisfaction survey [1]. However, this can be done through the study and investigation of raw drug materials, drug intermediates, final products, formulations, impurities, degradation products, and biological samples containing drugs and their metabolites to know the physicochemical properties even after their post-marketing [2]. Therefore, it is envisaged that proper corrective action should be needed at any phase of the study process to promote its safety and efficacy. Prominently, such investigation discloses the (i) effect of excipients on the drug formulation, (ii) incompatibility between excipient and drug, (iii) suitability of the excipient over drug administration to release the exact active component in the body, and (iv) capability of excipients in stabilizing the drug against degradation due to environmental factors [3].

Further, it is anticipated that, the research on discovery of new drugs are being lowering in recent days and the researchers and pharmaceutical companies are majorly focusing in preparation of drug modifications including active pharmaceutical ingredients (APIs), targeted to furnish the pharmacological activity with optimum therapeutic efficacy [4]. Evidently, it is important to mention here that nearly $40 \%$ of approved drugs and $90 \%$ of pipeline drugs are medications with poor solubility [5]. As an important property in view of pharmacological activity, drug molecules exhibited nearly 100-1000 times solubility, whereas cocrystals and polymorphs exhibited 4-20 times and 2-3 times, respectively. In addition, the solubility leads to the salt formation; a three component system consisted with acid, base, and solvent. However, the easiest approach to modify the properties of parent drug is salt formation where the unenviable features are conquered with ionizable functional groups. Cocrystals and APIs are non-ionizable neutral drugs, restricted with salt formation but fortunately the engineering of intermolecular interactions resulted in the generation of desired properties [6]. The solid drug substance that can be able to exist in more than one crystalline phase with various packing arrangements or molecular conformations in the same molecular species is considered as polymorphism [7]. It is estimated that, more than $50 \%$ of drugs exhibited polymorphism depending on the environmental conditions, and each form can significantly exhibited distinct physicochemical properties such as solubility, heat capacity, melting point, and sublimation point. Consequently, critical investigation on polymorphism of pharmaceutical solids during the pharmaceutical unit operations is worthful to alter the stability and bioavailability of the final drug product. Hence, it is recommended that 
proper monitoring of different solid-state forms and understanding the drug interactions with water gains paramount importance to ensure the high-quality product [8].

Different analytical techniques were reported in the literature to measure the structural differences of the solid pharmaceuticals such as X-ray diffraction, kinetic techniques, gravimetric, volumetric, electrochemical, spectroscopic, and various separation techniques [9-14]. Among various spectroscopic techniques, Raman spectroscopy has gained much attention and widely used technique for the identification of polymorphs due to non-invasiveness, small amount of sample requirements, and specificity. Besides, this technique is able to identify various physical forms of medicine such as liquid, powder, cream, tablet, and ointment, but is not useful for some medicines as they do not show Raman scattering. Inspired by this, here in this paper, we are compared the experimental results studied for the detection of polymorphism in Lamivudine and Finasteride drugs using Fourier transform (FT)Raman spectroscopy, to illustrate the advantages of the technique in the detection of polymorphism over other techniques. The IUPAC name of the Lamivudine (Fig. 1) is [cis-4-amino-1-(2-hydroxymethyl1,3-oxathiolan-5-yl)-(1H)-pyrimidin-2-one], which is a 1,3-oxathiolane nucleoside analog having antiviral activity and exists in at least two polymorphic forms. This drug is used in the medication to treat the HIVpositive and hepatitis B-positive patients. The two polymeric forms are needle-shaped crystals (Form I) and bipyramidal crystals (Form II) [15]. Similarly, Finasteride (Fig. 1) supplied under the trade name PROSCAR and the IUPAC name is $17 \beta$-(n-tert-butyl-carbamyl)-4-aza- $5 \alpha$-androst1 -en-3-one. It is a $5 \alpha$-reductase inhibitor used in the treatment of acne, female hirsutism, and prostatic hyperplasia. Finasteride also exhibited two polymorphic forms in which the orthorhombic crystal structure is Form I and the monoclinic crystal structure is Form II [16].

\section{METHODS}

The pure crystalline polymorphic forms of Lamivudine (Form I and Form II) and Finasteride (Form I and Form II) drugs were synthesized in APL Research Centre, Aurobindo Pharma Limited, Hyderabad, India.

\section{Preparation of polymorphic mixtures}

Initially, the pure forms of Lamivudine and Finasteride drugs were ground with the help of porcelain mortar and pestle. Later, polymorphic mixtures of Form I and Form II for both Lamivudine and Finasteride drugs were prepared with different weight ratios for the study $[15,16]$. In addition to the test sample, calibration standards were also prepared to compare the results and it was observed from the FT-IR data that the grinding process does not induce the polymorphism.

\section{Instrumentation}

Polymorphism in Finasteride drug was investigated using NXR FTRaman spectrophotometer, Thermoelectron Corporation, USA, with the slit aperture of $150 \mathrm{~mm}$ and the laser power of $1.0 \mathrm{~W}$. The $\mathrm{CaF}_{2}$ crystal as beam splitter with InGaAs detector with a 180-degree reflective configuration for sample position adjustment was used. A total of 512 scans at resolution of $4 \mathrm{~cm}^{-1}$ were acquired for each of standards and samples. Similarly for Lamivudine drug, Thermo Nicolet 5700 NXR-FT Raman spectrometer equipped with Nd-YAG laser source at a wavelength of $1064 \mathrm{~nm}$ was used. The InGaAs detector with 150 scans at $0.7 \mathrm{w}$ exciting power and $4 \mathrm{~cm}^{-1}$ spectral resolution and the scans were collected between 4000 and $200 \mathrm{~cm}^{-1}$. In addition, polarized light microscopy, FT-IR spectroscopy, differential scanning calorimeter, thermo gravimetric analysis, and X-ray powder diffraction studies were performed to further evidence the FT-Raman spectroscopic results.

\section{Preparation of sample for Raman spectroscopy}

A powdered sample was placed in a sample holder and it was a gold coated round shape central cavity and the cavity extended to other side of the holder. The holder was kept in such a way that the top face was placed over the stainless steel base and nearly $30 \mathrm{mg}$ of the sample was poured into the cavity. The sample was gently pressed with the finger press and was placed in the mount holds, which holds the sample at the correct height in the sample compartment. For tablet samples, the tablet was cut with a razor blade and placed in a spring action clamp holder in the sample compartment $[15,16]$.

\section{RESULTS}

To ensure that, the Raman spectroscopy is one of the best experimental techniques in detection and identification of polymorphic forms in drug substances and here, in this paper, we compared the Raman spectroscopic experimental results of Lamivudine and Finasteride drugs $[15,16]$. First of all, reference standards for Form I and Form II polymorphs of Lamivudine were thoroughly characterized by FT-IR spectroscopy, differential scanning calorimeter, thermo gravimetric analysis, and X-ray powder diffraction studies including Raman spectroscopy and confirmed that the purity of the polymorphs were $100 \%$. Raman spectrum of Form I showed a characteristic peak at $697 \mathrm{~cm}^{-1}$ and showed no peak at 463 and $798 \mathrm{~cm}^{-1}$ which further confirms the absence of Form II (Fig. 2).

Later, the drug mixture containing $6 \%(\mathrm{w} / \mathrm{w})$ was analyzed by grinding at various time intervals, that is, $5,10,15$, and 30 min to test the effect of grinding on polymorphism and was characterized by FTRaman spectroscopy. As shown in Fig. 3, all the above ground samples of different time intervals show the same peak intensity at $798 \mathrm{~cm}^{-1}$ Raman shift which confirmed that the grinding process does not induce polymorphic conversion for both polymorphic forms as well as for the mixture containing 6\% (w/w) of Form II in Form I. Therefore, there is no effect of grinding for the ground samples of polymorph standard mixtures, which were prepared with the help of porcelain mortar and pestle [15].

Again, FT-Raman spectra of both Form I, Form II, and mixture containing $4 \%(\mathrm{w} / \mathrm{w})$ of Form II were studied. The spectra showed different pattern, and observed a Raman band particularly at $798 \mathrm{~cm}^{-1}$ characteristic of Form II in polymorphic mixture containing 4\% (w/w) of Form II in Form I (Fig. 4).

In continuation, FT-Raman analysis was performed for the two polymorphic forms of Finasteride, as shown in Fig. 5, which divulges that the Raman shifts between the two polymorphic forms were clearly distinguishable. The Raman spectra of Finasteride Form I and Form II tablets prepared at APL and the Raman spectra of the placebo were<smiles>Nc1ccn([C@H]2CS[C@@H](CO)O2)c(=O)n1</smiles><smiles>CC(C)(C)NC(=O)[C@H]1CCC2C3CCC4NC(=O)C=CC4(C)C3CC[C@@]21C</smiles>

Fig. 1: Structure of lamivudine and Finasteride drugs 


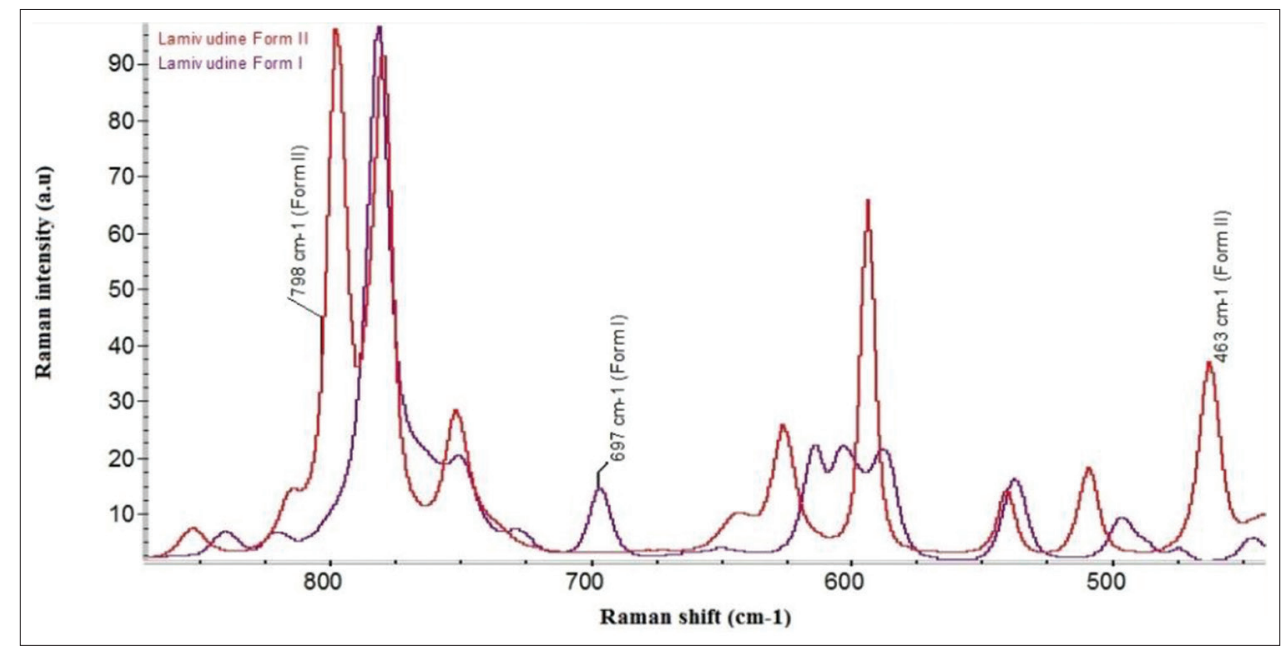

Fig. 2: Collation of lamivudine Forms I and II with Fourier-transform-Raman spectra

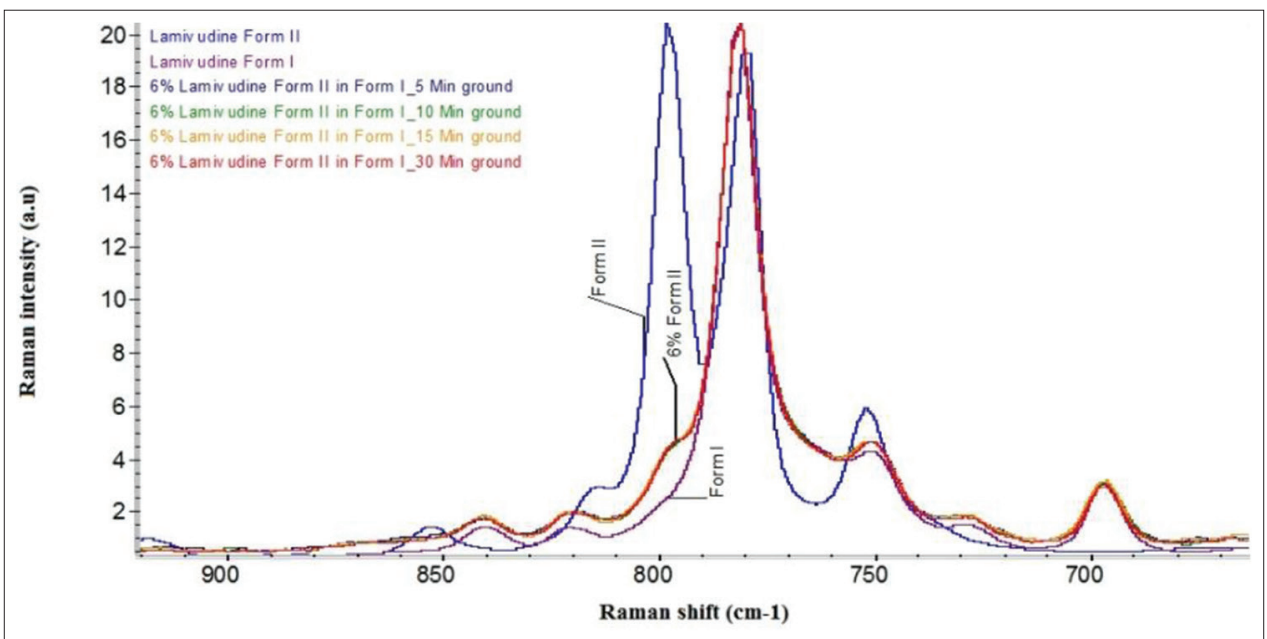

Fig. 3: Collation of lamivudine forms I and II and ground samples at various time intervals containing 6\% Form II with Fourier-transformRaman spectra

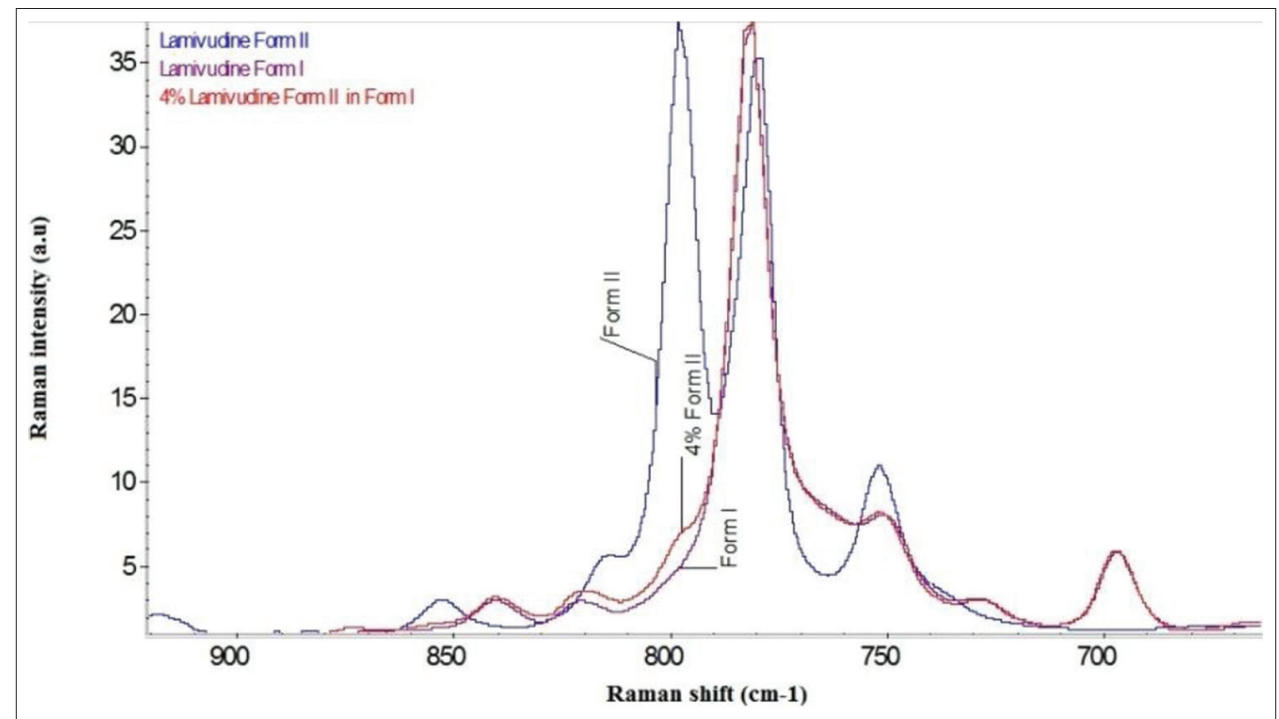

Fig. 4: Collation of Fourier-transform-Raman spectra of lamivudine forms I and II and 4\% (w/w) Form II

recorded (Fig. 6). The two polymorph forms showed a clear remarkable difference in the Raman shift region between 1750 and $1550 \mathrm{~cm}^{-1}$. It was observed that no placebo interference was noticed in this region and therefore this region is suitable for identification and detection of 


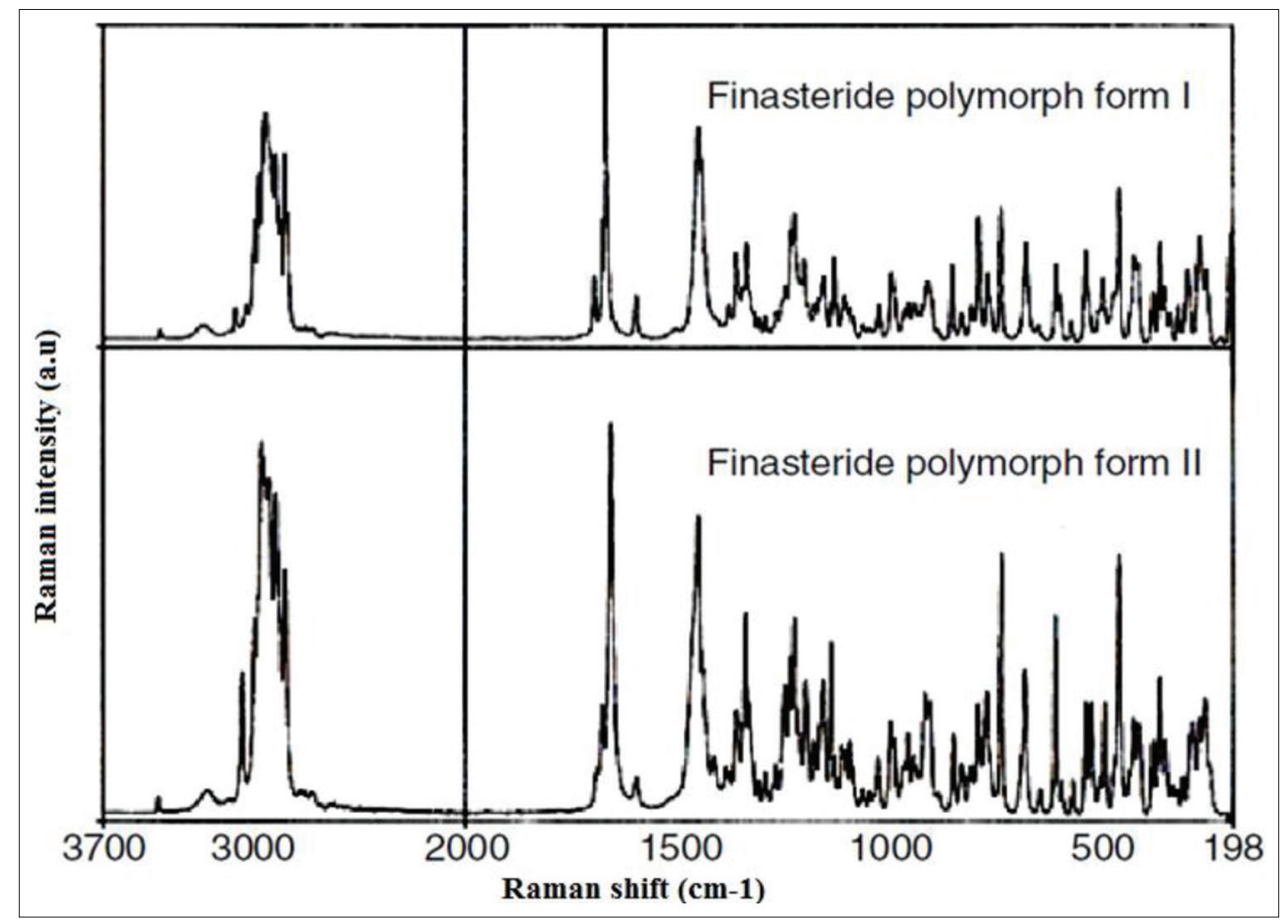

Fig. 5: Raman spectra of Finasteride polymorph Form I and Form II

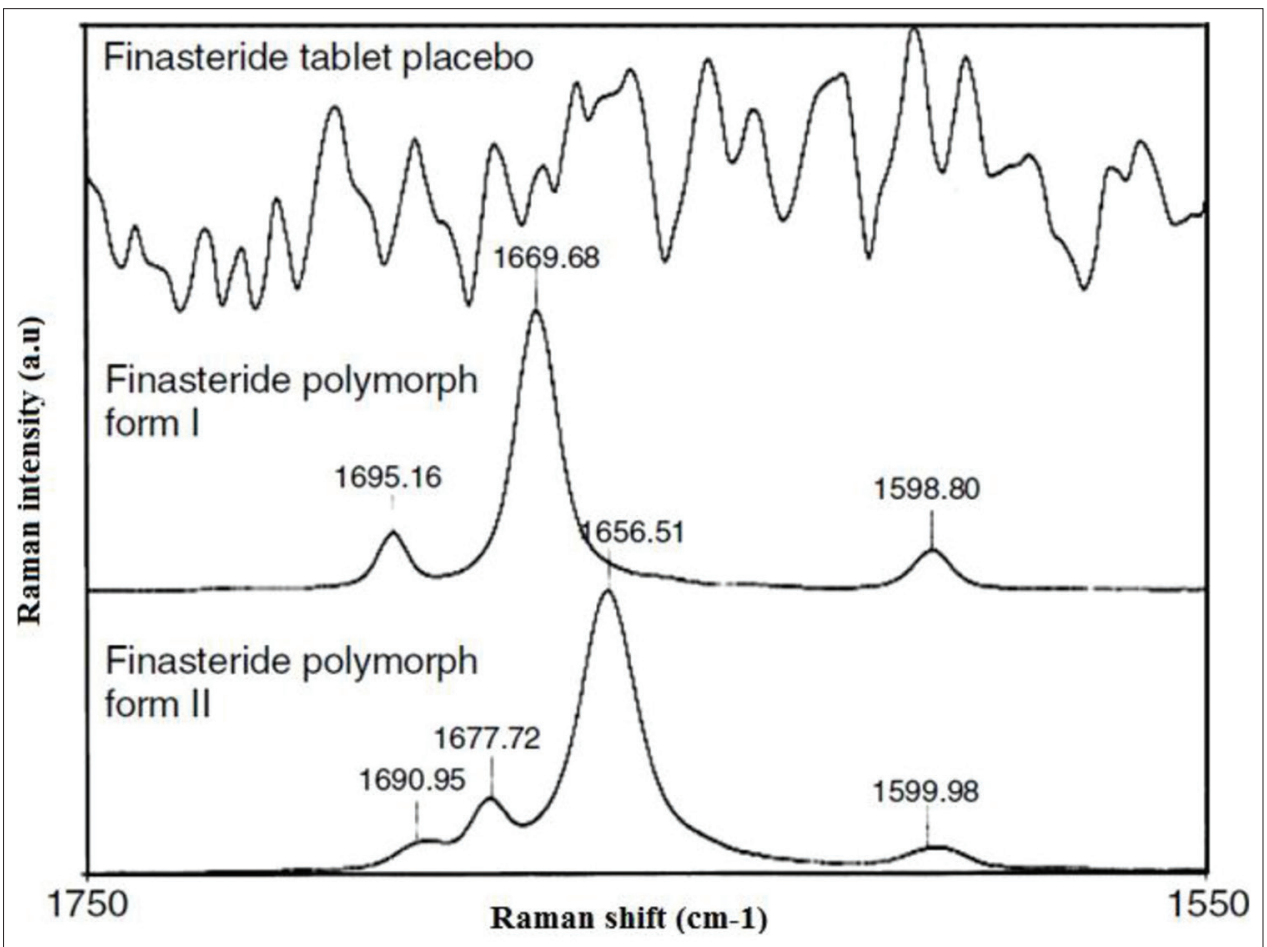

Fig. 6: Raman spectra of Finasteride polymorph Form I, Form II, and placebo in the quantification region

the polymorphic forms of Finasteride in the tablets. The characteristic peaks of the polymorphic Form I were present at 1670 and $1695 \mathrm{~cm}^{-1}$, and for polymorphic Form II at nearly 1656 and $1677 \mathrm{~cm}^{-1}$. The Raman spectral peak at $1598 \mathrm{~cm}^{-1}$ was common for Finasteride polymorphic forms and was clearly indicated that there was no interference from placebo in the region between 1750 and $1550 \mathrm{~cm}^{-1}$. The major characteristic peak of the polymorphic Form I was observed nearly at $1670 \mathrm{~cm}^{-1}$ and the minor peak nearly at $1695 \mathrm{~cm}^{-1}$. The major peak is used for detection of the polymorph Form I in Form II tablets. In the same way, the major characteristic peak of polymorphic Form II was observed at $1656 \mathrm{~cm}^{-1}$ and the minor peak at $1677 \mathrm{~cm}^{-1}$, simultaneously the major peak of Form II was used for detection of the polymorph Form II in Form I tablets [16].

After studying above Raman spectral patterns, FT-Raman spectroscopy was finalized for the quantification of Lamivudine as well as Finasteride drug substances, and the method was developed by the preparation of polymorphic mixtures. The advantages of Raman spectroscopy over other analytical techniques are (i) very little sample is required for analysis, (ii) ease of sample preparation, which includes the fact that 
both glass and water show very little Raman scattering, (iii) Raman spectra showed invariably sharp, well resolved bands compare to IR spectra due to the minor contribution of overtone vibrations in Raman spectra, resulting in much less broadening and a better resolution of bands compared to IR spectra [17], and (iv) Raman spectroscopy does not suffer from the sampling problems that are common in X-ray powder diffraction, where preferred orientation and specimen displacements are serious restrictions for the application of quantitative method [18].

\section{DISCUSSION}

The existence of the drugs in more than one solid form is termed as polymorphism, which could become one of the hot research focus in recent days to investigate the most stable and effective form of drug material for its quality assurance. Among several techniques, FT-Raman spectroscopy method is the best one for the detection of polymorphism in medicines and here we compared the results obtained for Lamivudine and Finasteride drugs $[15,16]$. Initially, X-ray powder diffractometry (XRPD) technique was used for the study, as it requires small quantity of drug sample and the sample is non-destructive during the identification and quantification of polymorphic forms. However, the technique has the limitation for unwanted polymorphic forms, as it do not exhibit distinct X-ray powder pattern and more intense characteristic peaks of unwanted polymorphic form of drug substance. In case of Lamivudine polymorphic forms, Form II was not having the distinct X-ray powder pattern and is not having the interference free regions, so it is difficult to quantify the Form II with low limit of detection by XRPD. Since, it is difficult to quantify the unwanted form with low limit of detection by XRPD [15].

Similarly, IR spectra of lamivudine forms I and II are well correlated with the previously published spectra and it was observed that polymorphic Form I exhibits a strong absorption band, but no bands were observed for polymorphic Form II [16]. Differential scanning calorimetry thermograms disclosed that the melting and crystallization temperature values of polymorphic forms of Lamivudine were different. As an advanced analytical method, FT-Raman spectroscopy has the advantage of direct investigation of solid samples without sample preparation. On investigation, Raman spectrum of Lamivudine Form I shows characteristic peak at $697 \mathrm{~cm}^{-1}$, as shown in Fig. 2. However, there were no such peaks observed at 463 and $798 \mathrm{~cm}^{-1}$ indicates the absence of Lamivudine Form II. Later, to reduce the source of error occurred due to the heterogeneity of the sample, pure polymorphic forms were ground in a porcelain mortar with the help of pestle for $5 \mathrm{~min}$ and were characterized by FT-Raman spectroscopy to identify the affect of grinding for polymorphic changes of pure polymorphs. Fig. 3 showed the FT-Raman spectra of Lamivudine Form II in Form I mixture containing $6 \%(\mathrm{w} / \mathrm{w})$ which was studied by grinding it at different time intervals of 5, 10, 15, and 30 min. Similarly, Fig. 4 showed the FT-Raman spectra of Form I, Form II and a mixture containing 4\% (w/w) of Form II, and Raman band observed at $798 \mathrm{~cm}^{-1}$ is the characteristic of Form II found in polymorphic mixture containing $4 \%$ (w/w) of Form II in Form I. It may be noted that this method used in this quantification work has been optimized in such a way that is found to be more sensitive to quantify Form II content in Lamivudine Form I leading to the lower detection levels [15].

Based on the above experimental results for Lamivudine Form II in Form I, FT-Raman spectroscopy was again used for the identification of Finasteride polymorphic mixtures [16]. It is observed from Fig. 5 that the Raman shifts between the two forms were clearly distinguishable. Later, the Raman spectra for Form I, Form II, and placebo were studied and shown in Fig. 6. The two polymorphic forms show a clear remarkable difference in the region between 1750 and $1550 \mathrm{~cm}^{-1}$. No placebo interference was observed in this region and therefore this region is suitable for identification and detection of the polymorphic forms of Finasteride. The characteristic peaks of the polymorph Form I were present at 1670 and $1695 \mathrm{~cm}^{-1}$ and for the polymorph Form II at about 1656 and $1677 \mathrm{~cm}^{-1}$. The peak at $1598 \mathrm{~cm}^{-1}$ was common for
Finasteride polymorphic forms [16]. This study showed that the FTRaman spectroscopic method is useful to detect the very low level of Finasteride polymorph Form II in polymorph Form I even though the drug concentration is less. This method might be employed for routine analysis for observing the polymorph changes during the formulation preparation and drug product.

There were several reports published for the identification and detection of polymorphic forms in pharmaceutical products using FT-Raman spectroscopy. To discuss few examples here, Roberts et al. studied the FT-Raman spectroscopic method for the quantification of a binary mixture containing beta and delta mannitol [19]. Usually, the polymorphic excipient of the mannitol is its beta form while the other alpha and delta polymorphs are the contaminants. Using this technique, the authors quantified up to the concentration levels down to $2 \%$ of the beta form. Another research group demonstrated the quantitative determination of polymorphic mixtures of calcium carbonate, that is, vaterite, aragonite, and calcite [20]. Amado et al. studied the vapor phase induced hydrate-anhydrate pseudopolymorphic transformations of theophylline crystals using a vibrational spectroscopic technique, that is, Raman spectroscopy [8]. Niclosamide is the antihelmintic as well as anti-cancer drug crystallizes into three solvated forms, that is, two monohydrates (NHa and $\mathrm{NHb}$ ) and one anhydrous (NAn) form [7]. Here, the authors evaluated and compared the application of Raman, near infrared (IR), and mid-IR spectroscopy in identification and quantification of the polymorphic forms of niclosamide. Besides, several reviews highlighted the advantages of Raman spectroscopic method in the analysis of polymorphism in various pharmaceuticals $[1,6]$.

\section{CONCLUSION}

Earlier days, the analysis of pharmaceuticals using Raman spectroscopy was limited because the technique had limitations with regard to sensitivity, fluorescence, and sub-sampling. Fortunately, these problems have been alleviated after its advancement through the development of FT-Raman spectrometer with charge coupled device, surface-enhanced Raman scattering, and transmission Raman geometry. Afterward, Raman spectroscopy has become notorious technique from the past three decades and has found incredible application in the analysis of environmental and biological samples, including pharmaceuticals. Further, it is important to mention here that the poor Raman scattering property of water makes the technique more superior than IR spectroscopy for the analysis of APIs in formulations especially when the drug concentration is low [1]. More importantly, the detection of polymorphism in the final dosage form using FT-Raman spectroscopic method is highly recommended due to (i) very little sample is required for analysis, (ii) ease of sample preparation, (iii) Raman spectra showed invariably sharp, well resolved bands, and (iv) Raman spectroscopy does not suffer from the sampling problems that are common in X-ray powder diffraction. Evidently, here in this paper, we have investigated and compared the polymorphism in Lamivudine and Finasteride drug substances using FT-Raman spectroscopic method at very low concentration level.

\section{ACKNOWLEDGMENT}

The authors gratefully acknowledge the Aurobindo Pharma Limited, Research Centre at Hyderabad for providing the analytical support to pursue this work.

\section{AUTHORS' CONTRIBUTIONS}

All the authors have contributed equally to this research work.

\section{CONFLICTS OF INTEREST}

The authors declare that they have no conflict of interest.

\section{AUTHORS' FUNDING}

This work is completely self-funded. 


\section{REFERENCES}

1. Melisew TA, Zebasil TM, Ellen M. Advances in surface-enhanced Raman spectroscopy for analysis of pharmaceuticals: A review. Vib Spectrosc 2018;98:50-63.

2. Bathish MA, Gazy AA, Jamal MK. Novel selective spectrophotometric methods for the determination of methimazole in pure form and in pharmaceutical formulation. Int J Pharm Pharm Sci 2020;12:62-9.

3. Bharate SS, Bharate SB, Bajaj AN. Incompatibilities of pharmaceutical excipients with active pharmaceutical ingredients: A comprehensive review. J Excip Food Chem 2010;1:3-26.

4. Priya CS. Design and evaluation of Lovastatin solid dispersions incorporated trilayer matrix tablets. Int $\mathrm{J}$ Pharm Sci Drug Res 2020;12:368-76.

5. Thenge R, Patel R, Kayande N, Mahajan N. Co-crystals of carvedilol: Preparation, characterization and evaluation. Int $\mathrm{J}$ App Pharm 2020;12:42-9.

6. Pindelska E, Sokal A, Kolodziejski W. Pharmaceutical cocrystals, salts and polymorphs: Advanced characterization techniques. Adv Drug Deliv Rev 2017;117:111-46.

7. Bhavana V, Chavan RB, Mannava MK, Nangia A, Shastri NR. Quantification of niclosamide polymorphic forms-a comparative study by Raman, NIR and MIR using chemometric techniques. Talanta 2019;199:679-88.

8. Amado A, Nolasco MM, Ribeiro-Claro PJ. Probing pseudopolymorphic transitions in pharmaceutical solids using Raman spectroscopy: Hydration and dehydration of theophylline. J Pharm Sci 2007;96:1366-79.

9. Suryanarayanan R. Determination of the relative amounts of $\alpha$-carbamazepine and $\beta$-carbamazepine in a mixture by powder $\mathrm{X}$-ray diffractometry. Powder Diff 1990;5:155-9.

10. Patel AD, Luner PE, Kemper MS. Quantitative analysis of polymorphs in binary and multi-component powder mixtures by near-infrared reflectance spectroscopy. Int J Pharm 2000;206:63-74.
11. Virtanen T, Maunu SL. Quantitation of a polymorphic mixture of an active pharmaceutical ingredient with solid state 13C CPMAS NMR spectroscopy. Int J Pharm 2010;394:18-25

12. Mrinalini CD, Pooja BK. Stability indicating HPTLC method for sofosbuvir and velpatasvir in combination. Int J Pharm Sci Drug Res 2020;12:129-35.

13. Khan $\mathrm{R}$, Irchhaiya $\mathrm{R}$. In vitro in vivo evaluation of niosomal formulation of famotidine. Int J Pharm Pharm Sci 2020;12:15-22.

14. Thorat Y, Shegaonkar A, Kumbhar S, Matole V, Hosmani A. Spectrophotometric determination of dasatinib in pharmaceutical formulations. Int J Curr Pharm Res 2020;12:68-71.

15. Rao MC, Reddy RC, Sekhar KB, Reddy YV. FT-Raman spectroscopy with chemometric methods for quantification of lamivudine form II in lamivudine form I. Asian J Chem 2017;29:1735-42.

16. Rao MC, Reddy RC, Sekhar KB, Reddy YV. Identification and detection of finasteride polymorphs in finasteride tablets by FT-Raman spectroscopy. Asian J Chem 2020;32:1865-8.

17. Zivkovic S, Momcilovic M, Staicu A, Mutic J, Trtica M, Savovic J. Spectrochemical analysis of powdered biological samples using transversely excited atmospheric carbon dioxide laser plasma excitation. Spectrochim Acta Part B 2017;128:22-9.

18. Croker DM, Hennigan MC, Maher A, Hu Y, Ryder AG, Hodnett BK. A comparative study of the use of powder X-ray diffraction, Raman and near infrared spectroscopy for quantification of binary polymorphic mixtures of piracetam. J Pharm Biomed Anal 2012;63:80-6.

19. Roberts SN, Williams AC, Grimsey IM, Booth SW. Quantitative analysis of mannitol polymorphs. FT-Raman spectroscopy. J Pharm Biomed Anal 2002;28:1135-47.

20. Dandeu A, Humbert B, Carteret C, Muhr H, Plasari E, Bossoutrot JM. Raman spectroscopy-a powerful tool for the quantitative determination of the composition of polymorph mixtures: Application to $\mathrm{CaCO}_{3}$ polymorph mixtures. Chem Eng Technol 2006;29:221-5. 